\title{
ANÁlise dA PERCEPÇÃO DOS ALUNOS SOBRE A QUALIDADE DA ÁGUA DOS BEBEDOUROS EM ESCOLAS DO MUNICÍPIO DE CABEDELO - PB
}

\author{
R. A. MELO ${ }^{1}$, T. K. S. SANTOS ${ }^{1}$, F. P. B. F. CARDOSO ${ }^{2}$, V.M. SILVA ${ }^{3}$, H. L. SILVA- \\ NETA $^{4}$, R. OLIVEIRA ${ }^{5}$
}

${ }^{1}$ Universidade Estadual da Paraíba ${ }^{1}$,

${ }^{2}$ Universidade Federal da Paraíba, Hospital Universitário Lauro Wanderley, Unidade de Farmácia Clínica

${ }^{3}$ Universidade Federal da Paraíba, Centro de Ciências da Saúde, Núcleo de Saúde Coletiva

${ }^{4}$ Universidade Federal de Pernambuco, Núcleo de Pesquisa em Inovação Terapêutica Suely Galdino, Laboratório de Imunomodulação e Novas Abordagens Terapêuticas

${ }^{5}$ Universidade Estadual da Paraíba, Departamento de Engenharia Sanitária e Ambiental do Centro de Ciências e Tecnologia.

E-mail para contato: silvanetahlj@gmail.com

RESUMO - Crianças e adolescentes passam um tempo considerável do dia no ambiente escolar, e que nesses ambientes a disponibilização de água ocorre geralmente por meio de bebedouros. Este trabalho visou avaliar a percepção dos alunos sobre a qualidade da água, limpeza e conservação dos bebedouros em um ambiente escolar. Para avaliação das respostas obtidas por meio dos questionários foi feita a categorização das respostas e codificação dos dados. Foi aplicada a análise estatística descritiva para resumir as médias e proporções de cada variável e análise de qui-quadrado para comparação de dados qualitativos. Essa investigação sobre a percepção dos alunos possibilitou a identificação de que o bebedouro é usado com frequência considerável, apesar de percepções negativas quanto à qualidade e segurança da água, bem como em relação à limpeza e conservação do equipamento.

Palavras-Chave: Ambiente escolar. Alunos. Bebedouros.

ABSTRACT - Children and adolescents spend considerable time during the day in the school environment, and in these environments water availability usually occurs through drinking fountains. This work aimed to evaluate students' perceptions about water quality, cleaning and conservation of drinking fountains in a school environment. For the evaluation of the answers obtained through the questionnaires the categorization of the answers and codification of the data was done. Descriptive statistical analysis was applied to summarize the means and proportions of each variable and chi-square analysis to compare qualitative data. This investigation of student perceptions made it possible to identify that the drinking fountain is used with considerable frequency, despite negative perceptions regarding water quality and safety, as well as the cleaning and conservation of the equipment. 


\section{INTRODUÇÃO}

A água é um recurso da natureza fundamental para a vida. No corpo humano, ela corresponde a maior proporção da massa corpórea e atua nos mais diversos processos fisiológicos e bioquímicos fundamentais para o crescimento, desenvolvimento, manutenção da vida e prevenção de diversas doenças. Suas funções cruciais incluem o transporte de nutrientes vitais e a eliminação de toxinas, que são indispensáveis para a vida (HUMPHREYS, et al, 2017; LENINGHER, 2014)

Dada sua relevância, a reposição oral de água é necessária uma vez que a mesma vai sendo perdida de acordo com o funcionamento do organismo. O Instituto de Medicina Norte Americano estabeleceu o nível adequado de ingestão de água para meninas adolescentes de 2,1 L por dia, já para meninos é de 2,4 L (Institute of Medicine, 2015). Pesquisas realizadas nos Estados Unidos da América (EUA) relatam baixa ingestão de água entre os adolescentes, com média de menos de três copos por dia, apesar da disponibilidade de água encanada em grande parte dos ambientes (PATEL et al., 2014a; SEBASTIAN, WILKINSONS, GOLDMAN, 2015).

As escolas, locais onde crianças e adolescentes passam a maior parte do dia, podem representar um cenário ideal para aumentar a ingestão de água (PATEL et al., 2010). E, como benefícios, o aumento da ingestão de água nas escolas pode possibilitar a manutenção do peso saudável, redução das cáries dentárias e melhoria na prontidão para a aprendizagem (D'ANCI, CONSTANT, ROSENBERG, 2006; EDMONDS CJ, JEFFES, 2009; POPKIN, D'ANCI, ROSENBERG, 2010). Estes estudos também sugerem que a ingestão de água pelos alunos nas escolas poderia melhorar a função cognitiva deles.

Em grande parte das escolas do Brasil, como ocorre no município de Cabedelo no Estado da Paraíba, a água potável é disponibilizada por meio de bebedouros. No Brasil, têm sido desenvolvidas pesquisas que buscam monitorar a qualidade da água de bebedouros em escolas, no entanto, poucos estudos consideram a percepção dos alunos acerca dos bebedouros e da água potável disponibilizada. Nos Estados Unidos, os estudos sugerem que alunos não utilizam os bebedouros por considerar a água não palatável ou não segura, ou considerar estes equipamentos sujos (ONUFRAK et al., 2014; PATEL et al., 2014A; PATEL et al., 2014b).

A exigência da qualidade da água objetiva a proteção à saúde. E, os critérios utilizados para garantir esta qualidade possibilitam o desenvolvimento de ações que garantam a segurança da água fornecida, minimizando a concentração ou eliminando os constituintes perigosos à saúde (D’AGUILA et al., 2000).

Desta maneira, a percepção dos alunos quanto aos bebedouros escolares foi investigada, visto que esta poderia estar relacionada com as intenções dos alunos em ingerir água quando na escola.

\section{MATERIAIS E MÉTODOS}

Com o objetivo de estudar a percepção dos alunos acerca dos bebedouros instalados nas escolas e quais as atitudes destes quanto à ingestão de água no decorrer do dia letivo, foram aplicados 100 questionários em 4 escolas do município de Cabedelo 
localizado no estado da Paraíba - Brasil, durante o período de outubro a novembro de 2015.

Para avaliar a ingestão de água dos alunos por dia foi perguntado quantos copos de água eles bebiam diariamente, tendo como base um copo de $300 \mathrm{~mL}$. Para investigar sobre o uso do bebedouro foi questionado quantas vezes por dia o bebedouro escolar era utilizado. Para confirmação do uso do bebedouro os alunos responderam "concordo totalmente", "concordo", "Não concordo, nem discordo", "discordo totalmente" às afirmações "Tomo a quantidade suficiente de água na escola" e, "Na escola, apenas tomo água que trago de minha casa". Este padrão de respostas teve como base a escala de Likert.

Fazendo uso desta mesma escala de satisfação, com o objetivo de investigar a percepção dos alunos sobre a qualidade da água, foram empregadas as afirmações "tomar água do bebedouro da escola pode me fazer adoecer", "confio na qualidade da água do bebedouro da escola", "a água do bebedouro da escola é limpa e segura", "o gosto e odor da água do bebedouro da escola são agradáveis", e, "a temperatura da água do bebedouro é agradável".

Para avaliar a percepção do aluno a respeito da quantidade e aspecto físico dos bebedouros, as afirmações utilizadas foram "a escola possui quantidade suficiente de bebedouros", "o bebedouro está sempre limpo", "o bebedouro encontra-se bem conservado". As respostas obtidas para a escala de satisfação foram posteriormente agrupadas nas categorias percepção positiva, neutra ou negativa de acordo com a afirmação. $\mathrm{O}$ gênero e a idade do aluno também foram incluídos no questionário.

Para montagem do banco de dados, foi determinado que cada pergunta corresponde a uma variável, houve a categorização das respostas e codificação dos dados (Rocha et al., 2006). Foi aplicada a análise estatística descritiva para resumir as médias e proporções de cada variável e análise de qui-quadrado para comparação de dados qualitativos.

Para o cálculo do tamanho da amostra, foi considerado o quantitativo de 4979 alunos matriculados nas 22 escolas municipais de ensino fundamental. $\mathrm{O}$ cálculo foi realizado por meio das Equações (1) e (2) (Barbeta, 2002), sendo n o tamanho da amostra procurada; N o tamanho da população (4979 alunos); $\mathrm{E}_{0}$ o erro amostral tolerável, adotado como $10 \%$; e $\mathrm{n}_{0}$ a primeira aproximação do tamanho da amostra.

$$
\begin{array}{ll}
\mathrm{n}=\mathrm{N} . \mathrm{n} 0 \mathrm{~N}+\mathrm{n} 0 & \text { EQ 1 } \\
\mathrm{n} 0=1 \mathrm{E} 02 & \text { EQ 2 }
\end{array}
$$

A estatística descritiva foi utilizada para resumir as proporções obtidas para as variáveis analisadas. E, também foi examinada a associação bivariada entre a percepção dos bebedouros e a ingestão de água na escola, por meio do programa IBM SPSS Statistics 21 .

\section{RESULTADOS E DISCUSSÃO}

O questionário foi aplicado a 100 alunos, 35 do gênero masculino e 65 do gênero feminino, a idade média dos alunos foi de 13,6 anos. Apesar de diversas respostas indicarem uma percepção negativa quanto aos bebedouros, à qualidade da água e ao aspecto físico do equipamento, $41 \%$ dos alunos relataram que utilizavam o bebedouro 2 vezes ao dia.

Em relação à qualidade da água, grande parte avaliou que o gosto e o odor (39\%), e a temperatura (44\%) são desagradáveis. A indicação de um bebedouro sujo (55\%), mal 
conservado (54\%), a associação da água do bebedouro com doenças (49\%), e a falta de confiança na qualidade da água (41\%) foram preponderantes.

Quando perguntados sobre a ingestão de água na escola, 32\% afirmaram tomar a quantidade de água necessária, já $40 \%$ responderam que não e $46 \%$ relataram não trazer água de casa.

Considerando a ingestão diária de água, as alunas $(46,2 \%)$ e alunos $(62,9 \%)$ responderam predominantemente que bebiam 4 ou mais copos de água por dia (Tabela 1). A maioria das alunas $(33,8)$ e dos alunos $(54,3)$ responderam que fazem uso 2 ou 3 vezes do bebedouro por dia (Tabela 2).

Tabela 1: Quantidade de copos de água por dia por gênero.

\begin{tabular}{|c|c|c|c|c|c|c|c|}
\hline Gênero & & \multicolumn{6}{|c|}{ Copos de água por dia, $\%$} \\
\hline & $\mathbf{N}$ & $\begin{array}{l}<1 \\
\text { copo }\end{array}$ & $\begin{array}{l}1 \text { copo de } \\
\text { água }\end{array}$ & $\begin{array}{l}2 \text { copos de } \\
\text { água }\end{array}$ & $\begin{array}{l}3 \text { copos de } \\
\text { água }\end{array}$ & $\begin{array}{l}4 \text { ou mais } \\
\text { copos de água }\end{array}$ & $\begin{array}{l}X^{2} \\
P \\
\text { valor }\end{array}$ \\
\hline Feminino & 65 & 7,7 & 6,2 & 12,3 & 27,7 & 46,2 & 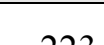 \\
\hline Masculino & 35 & 5,7 & 5,7 & 0 & 25,7 & 62,9 & , 223 \\
\hline
\end{tabular}

Tabela 2: Utilização do bebedouro por dia por gênero.

\begin{tabular}{|l|c|c|c|c|c|c|}
\hline Gênero & \multicolumn{6}{|c|}{ Utilização do bebedouro por dia, \% } \\
\hline & N & Não utiliza & 1 vez & 2 ou 3 vezes & 4 vezes ou mais & $\begin{array}{c}\mathbf{X}^{\mathbf{2}} \\
\text { p- valor }\end{array}$ \\
\hline Feminino & 65 & 23,1 & 26,2 & 33,8 & 16,9 & \multirow{2}{*}{, 199} \\
\hline Masculino & 35 & 14,1 & 14,3 & 54,3 & 17,1 & \\
\hline
\end{tabular}

Tabela 3: Associação entre utilização do bebedouro e percepções positivas, neutras e negativas acerca da água e bebedouro.

\begin{tabular}{|c|c|c|c|c|c|c|c|}
\hline \multirow[b]{2}{*}{ Questionamento } & \multirow[b]{2}{*}{ Percepção } & & \multicolumn{4}{|c|}{ Uso do Bebedouro, \% } & \multirow[b]{2}{*}{$\begin{array}{c}\mathbf{X}^{2} \\
\mathbf{p}- \\
\text { valor }\end{array}$} \\
\hline & & $\mathbf{N}$ & $\begin{array}{l}\text { Não } \\
\text { usa }\end{array}$ & $\begin{array}{c}1 \\
\text { vez }\end{array}$ & $\begin{array}{l}2 \text { ou } 3 \\
\text { vezes }\end{array}$ & $\begin{array}{c}4 \text { ou } \\
\text { mais } \\
\text { vezes }\end{array}$ & \\
\hline \multirow{3}{*}{$\begin{array}{c}\text { A água do bebedouro é } \\
\text { limpa e segura }\end{array}$} & Positiva & 30 & 10 & 18,2 & 41,5 & 41,2 & \multirow{3}{*}{,052 } \\
\hline & Neutra & 36 & 40 & 31,8 & 39,0 & 29,4 & \\
\hline & Negativa & 34 & 50 & 50 & 19,5 & 29,4 & \\
\hline \multirow{3}{*}{$\begin{array}{l}\text { Confiança na qualidade } \\
\text { da água do bebedouro }\end{array}$} & Positiva & 23 & 15 & 9,1 & 27,5 & 43,8 & \multirow{3}{*}{,019 } \\
\hline & Neutra & 35 & 20 & 36,4 & 40,0 & 43,8 & \\
\hline & Negativa & 40 & 65 & 54,5 & 32,5 & 12,5 & \\
\hline \multirow{3}{*}{$\begin{array}{l}\text { Associação da água do } \\
\text { bebedouro com doenças }\end{array}$} & Positiva & 8 & 0 & 4,5 & 15,0 & 5,9 & \multirow{3}{*}{,016 } \\
\hline & Neutra & 42,4 & 45 & 18,2 & 55,0 & 41,2 & \\
\hline & Negativa & 49,5 & 55 & 77,3 & 30,0 & 52,9 & \\
\hline \multirow{3}{*}{$\begin{array}{l}\text { O bebedouro encontra-se } \\
\text { bem conservado }\end{array}$} & Positiva & 20 & 20 & 18,2 & 24,4 & 11,8 & \multirow{3}{*}{,239 } \\
\hline & Neutra & 26 & 10 & 22,7 & 36,6 & 23,4 & \\
\hline & Negativa & 54 & 70 & 59,1 & 39,0 & 64,7 & \\
\hline \multirow{3}{*}{$\begin{array}{l}\text { O bebedouro está sempre } \\
\text { limpo }\end{array}$} & Positiva & 20 & 20 & 13,6 & 22,0 & 23,5 & \multirow{3}{*}{, 517} \\
\hline & Neutra & 25 & 15 & 18,3 & 34,1 & 23,5 & \\
\hline & Negativa & 55 & 65 & 68,2 & 43,9 & 52,9 & \\
\hline
\end{tabular}


Análises de correlação possibilitam o conhecimento sobre a percepção dos alunos a respeito dos bebedouros e se esta está associada com o uso desses equipamentos, e, consequentemente com a ingestão de água na escola. Neste levantamento, o relato de não uso do bebedouro esteve relacionado com a não confiança na qualidade da água, já os relatos de uso estiveram associados com percepções positivas ou neutras em relação a qualidade da água (Tabela 3). Estudos realizados nos EUA relatam que alunos não utilizam os bebedouros escolares por falta de atratividade do equipamento e por preocupações com a segurança da água (PATEL et al.; 2010; ONUFRAK, 2014; PATEL et al., 2014a).

Esta preocupação com a segurança da água também é percebida nos alunos que afirmaram não fazerem uso do bebedouro, já que estes não consideram a água limpa e segura $(50 \%)$, não confiam na qualidade $(65 \%)$, e, associam a água à transmissão de doenças (55\%). Entretanto, mesmo para os alunos que fazem uso dos bebedouros, esta preocupação é presente. Mesmo em circunstâncias em que a água da torneira é segura, a preocupação com sua qualidade a água pode torná-la não atrativa (PATEL et al., 2014a). A percepção negativa de familiares acerca da água de torneira poderia influenciar a decisão dos alunos em bebê-la (PATEL et al.; 2010). Realizar análises da água para contaminantes e comunicar os resultados dessas análises para a comunidade escolar, pode ajudar a diminuir a preocupação dos alunos quanto à segurança da água dos bebedouros (PATEL et al., 2014b). E, em casos de descoberta da não potabilidade da água, a escola pode providenciar outro meio de fornecer água aos alunos.

A ausência de opinião quando abordados os conceitos de água limpa, segura e a associação da água com doenças, pode retratar desconhecimento sobre esses conceitos e acerca das características que estão relacionadas com a qualidade da água, gerando insegurança em emitir opinião sobre a questão.

A percepção negativa em relação ao aspecto físico dos bebedouros foi preponderante entre os alunos. Mesmo os que utilizam os bebedouros, classificaram esses equipamentos como sujos e não conservados, demonstrando a necessidade de maior preocupação da gestão escolar com a limpeza e manutenção desses.

Nesta análise, apesar da observação de percepção negativa dos alunos em relação à segurança da água e estrutura física do bebedouro, a utilização desse equipamento é notória. Pode-se ressaltar que o bebedouro, nas escolas avaliadas, é o principal e permanente meio de disponibilização de água potável para os alunos. Dessa forma, a limpeza e manutenção desses equipamentos são imprescindíveis, bem como o planejamento de ações que abordem conceitos e questões relacionados à qualidade e segurança da água, para que os alunos construam percepções mais positivas. A criação de um sistema interno de acompanhamento e monitoramento da água consumida, a produção de materiais institucionais que visem promover o conhecimento sobre o consumo e ingestão de água representam ações que podem colaborar com esse processo (COBAN, 2011; SILVA et al., 2015).

\section{CONCLUSÃO}

A aplicação de questionário com alunos possibilitou a identificação de que o bebedouro é usado com frequência considerável, apesar de percepções negativas quanto à qualidade e segurança da água, bem como em relação à limpeza e conservação do equipamento. No entanto, apesar de métodos qualitativos possibilitarem o desenvolvimento de hipóteses e a investigação da percepção dos alunos a respeito da água e bebedouro escolar, os resultados não podem ser generalizados. Novos estudos a serem 
realizados com amostras representativas de escolas podem expandir as discussões sobre os resultados observados.

As percepções dos alunos poderiam ser trabalhadas a partir do planejamento de projetos pela gestão escolar que abordem a segurança e a qualidade da água, possibilitando a construção de novos conhecimentos acerca do consumo e ingestão de água.

\section{REFERÊNCIAS}

BARBETTA PA. Estatística aplicada as ciências sociais. $5^{\mathrm{a}}$ ed. Santa Catarina: Editora UFSC; 2002.

COBAN, G U; AKPINAR, E; KUCUKCANKURTARAN, E; YILDIZ, E; ERGIN, O. Elementary school students' water awareness. International Research in Geographical \& Environmental Education, v. 20, n. 1, p.65-84, 2011.

D'AGUILA, P. S. de; CRUZ ROQUE, O. C. de; MIRANDA, C. A. S.; FERREIRA, A. P. Avaliação da qualidade de água para abastecimento público do Município de Nova Iguaçu. Cad. Saúde Pública, Rio de janeiro. 16 (3): 791-798, Jul-Set, 2000.

D'ANCI KE, CONSTANT F, ROSENBERG IH. Hydration and cognitive function in children. Nutrition Review, v. 10, n.64, p. 457-464, 2006.

EDMONDS CJ, JEFFES B. Does having a drink help you think? 6-7-year-old children show improvements in cognitive performence from baseline to test after having a drink of water. Appetite, v.3, n.52, p.469-472, 2009

HUMPHREYS, K; VADHER, D; ALLEN, Y; PATEL, N; SHAH, S. Examining the links between hydration, nutrition and mouth health. British Journal of Nursing, v. 9, n. 10, p. 566-570, 2017.

Institute of Medicine. Dietary reference intakes: water, potassium, sodium, chloride, and sulfate. Disponível em: http://www.iom.edu/REports/2004/Dietary-Reference-IntakesWater-Potassium-Sodium-Chloride-and-Sulfate.aspx. Acessado em: Novembro de 2015.

LEHNINGER, A.L. Princípios de Bioquímica, 6ª Ed., Artmed, São Paulo, SP, 2014.

ONUFRAK SJ, PARK S, SHARKEY JR, MERLO C, DEAN WR, SHERRY BL. Perceptions of tap water and school water fountains and association with intake of plain water and sugar-sweetened beverages. Journal of School Health, n. 84, p.195-204, 2014

PATEL AI, et al. Middle School student attitudes about school drinking fountains an water intake. Academic pediatrics, v. 5, n. 14, p.471-7, 2014. (a).

PATEL AI, HECHT K, HAMPTON KE, GRUMBACH JM, BRAFF-GUAJARDO E, BRINDIS CD. Tapping into water: key considerations for achiving excellence in school drinking water acess. American journal of Public Health, v. 7, n.104, p.1314-1319, 2014. (b). 
SEBASTIAN RS, WILKINSONS EC, GOLDMAN JD. Drinking water intake in the US: what we eat in America, NHANES 2005-2008. Food Surveys Research Group Dietary Data Brif No. 7. Disponível em: http://www.ars.usda.gov/Services/docs.htm?docid+19476. Acessado em: Novembro de 2015.

SILVA, J T; SILVA, B B S; SILVA, A A; SILVA, G N R. Análise físico-química da qualidade de água nos bebedouros da escola e conscietização dos estudantes sobre seu consumo. IICONEDU - Congresso Nacional de Educação, Campina Grande - PB, Brasil, 2015 . 